\title{
Comparative evaluation of two reconstructive methods following laparoscopic assisted subtotal gastrectomy in dogs
}

\author{
Jalal Bakhtiari ${ }^{1,4^{*}}$, Mahbobeh Abdi ${ }^{1}$, Alireza R Khalaj ${ }^{2}$, Farzad Asadi ${ }^{3}$ and Amir Niasari-Naslaji ${ }^{1}$
}

\begin{abstract}
Background: Laparoscopic gastrectomy is a new and technically challenging surgical procedure with potential benefit. The objective of this study was to investigate clinical and para-clinical consequences following Roux-en-Y and Jejunal Loop interposition reconstructive techniques for subtotal gastrectomy using laparoscopic assisted surgery.

Results: Following resection of the stomach attachments through a laparoscopic approach, stomach was removed and reconstruction was performed with either standard Roux-en-Y $(n=5)$ or Jejunal Loop interposition $(n=5)$ methods. Weight changes were monitored on a daily basis and blood samples were collected on Days 0,7 and 21 post surgery. A fecal sample was collected on Day 28 after surgery to evaluate fat content. One month post surgery, positive contrast radiography was conducted at 5, 10,20, 40,60 and 90 minutes after oral administration of barium sulfate, to evaluate the postoperative complications. There was a gradual decline in body weight in both experimental groups after surgery $(P<0.05)$. There was no difference in blood parameters at any time after surgery between the two methods $(P>0.05)$. Fecal fat content increased in the Roux-en-Y compared to the Jejunal loop interposition technique $(P<0.05)$. No major complications were found in radiographs and gastric emptying time was similar between the two groups $(P>0.05)$.

Conclusion: Roux-en-Y and Jejunal loop interposition techniques might be considered as suitable approaches for reconstructing gastro-intestinal tract following gastrectomy in dogs. The results of this study warrant further investigation with a larger number of animals.
\end{abstract}

Keywords: Gastrectomy, Laparoscopy, Reconstruction, Dog

\section{Background}

Several approaches of open surgeries including Roux en $Y$ (gastrojejunostomy), Billroth I and jejunal loop interposition (jejunoduodenostomy) and Billroth II (gastrojejunal anastomosis) were used to reconstruct partial or total gastrectomy [1-4]. Comparing Roux en Y and Billroth II procedures, the Roux-en-Y approach showed less reflux symptoms and less chronic fundic atrophic gastritis [5]. Although Roux-en-Y is the most common method of reconstruction for total gastrectomy $[1,6]$, it is associated with abdominal pain, nausea, vomiting, fullness

\footnotetext{
*Correspondence: bakhtiar@ut.ac.ir

${ }^{1}$ Faculty of Veterinary Medicine, University of Tehran, Tehran, Iran ${ }^{4}$ Department of Surgery and Radiology, Faculty of Veterinary Medicine, University of Tehran, P. O. Box: 14155-6453, Tehran, Iran

Full list of author information is available at the end of the article
}

and morbidity, as high as 30\% [7-10]. Furthermore, due to the lack of food passage through the duodenum, the potential benefit of the duodenum for normal digestion is disrupted following Roux-en-Y reconstructive technique, $[11,12]$. On the contrary, in Jejunal loop interposition, the food passage through the duodenum was maintained [6-8,13]. Regardless, there were no differences between Roux-en-Y and Jejunal Loop interposition in prospective quality of life, and no complications in long term studies [14-16].

Laparoscopic assisted surgery has several advantages compared with open surgery including reduced blood loss, less postoperative pain, better oral intake, earlier bowel function recovery, shorter time of hospital stay and reduced risk of sepsis [17]. The laparoscopic assisted procedure is less invasive than conventional open gastrectomy. 
It leads to quick recovery of gastrointestinal functions and reduces pain [18]. More recently, we have demonstrated that laparoscopic assisted Roux-en-Y reconstructive technique had less post-operative complications compared to open surgery in dogs [19]. Laparoscopic assisted subtotal gastrectomy is a feasible and safe alternative approach compared with an open surgery [20,21]. In the present study, laparoscopic assisted subtotal gastrectomy was conducted using Roux-en-Y or Jejunal Loop interposition reconstructive techniques in dogs ( $n=5$ in each group).

\section{Methods}

The present study received an approval by the Animal Ethics Committee of the Faculty of Veterinary Medicine, University of Tehran (BNS498/20.05.08). The experiment was conducted at Small Animal Veterinary Medicine Hospital, Faculty of Veterinary Medicine, University of Tehran. Ten healthy mixed-breed male dogs $(26.5 \pm 1.99 \mathrm{Kg}$ of weight; $3.2 \pm 0.29$ years of age) were selected among homeless animals by permission from the animal shelter. All dogs received rabies vaccination and anti-parasitic agents (Praziquantel $5 \mathrm{mg} / \mathrm{kg}$ and piperazine $100 \mathrm{mg} / \mathrm{kg}, \mathrm{p}$. o). Prior to the surgery, the experimental dogs received a balanced diet with free access to water. Following surgery, food and water restriction were implemented while dogs received Ringer-Lactate serum for three days subsequent with the balanced soft and/or syrupy diet supplemented with minerals and vitamins.

Under general anesthesia (Acepromazine $0.04 \mathrm{mg} / \mathrm{kg}$, IM; Tiopental-sodium $10 \mathrm{mg} / \mathrm{kg}$, IV; Halothane, 1-1.5\%) laparoscopic assisted gastrectomy was performed with three laparoscopic portals. The abdomen was insufflated with carbon dioxide $(\mathrm{P}=14 \mathrm{mmHg})$. Following insertion of the first trocar $(10 \mathrm{~mm})$ at the umbilicus, the laparoscopic telescope (0 degree; $10 \mathrm{~mm}$ ) was introduced into the abdominal cavity. The other two trocars $(5 \mathrm{~mm})$ were placed in 1/3 upper left and right midline. Gastric vessels were clipped and cut by clip-applicator and bipolar cautery, respectively. The gastroepiploic vessels and its branches were resected after coagulation with bipolar electrocuater forceps. After resecting stomach attachments, a $5 \mathrm{~cm}$ incision was performed on the place of the first laparoscopic trocar and four fifth of the distal stomach was removed (Figure 1). After gasterectomy, dogs were assigned randomly into two reconstructive techniques ( $\mathrm{n}=5$ in each group). In Roux-en-Y group, the cutting edge of duodenum was closed with two layer inverting suture by hand sewing. Then the jejunum was cut from the $20 \mathrm{~cm}$ distal of the Treitz ligament. The distal part of the cutting edge of the jejunum was anastomosed to the remaining part of the stomach and the proximal part was anastomosed to the rest of the jejunum using the end to side pattern suturing by hand sewing (Figure 2). In the Jejunal Loop interposition

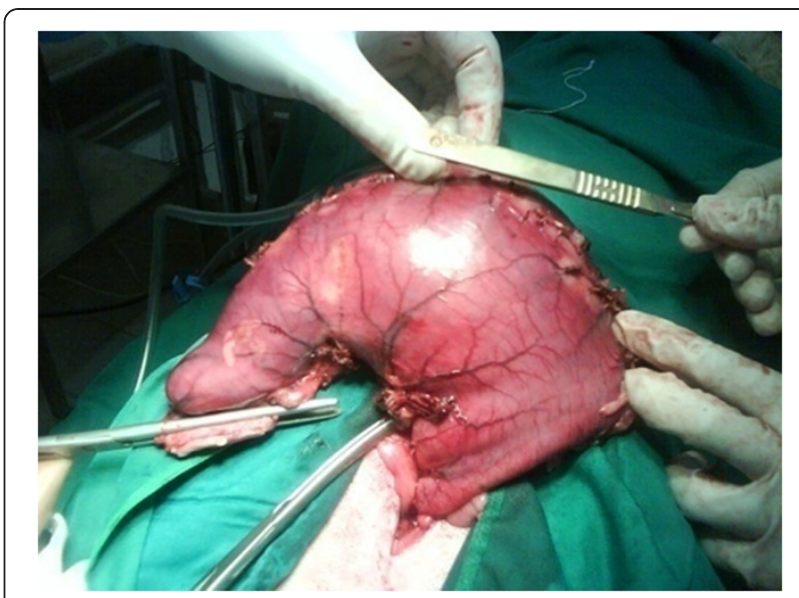

Figure 1 The stomach was removed from mini-laparotomy incision.

group, the reconstructive technique was performed with the resection of $20 \mathrm{~cm}$ length of jejunum from the $20 \mathrm{~cm}$ distal to Treitz ligament. Then, two ends to side anastomosis were applied between the remaining part of the stomach and proximal part of the jejunum and between the distal part of the jejunum and duodenum (Figure 3). Finally the abdomen was lavaged and closed in a routine manner. The food restriction was continued for three days after surgery. Experimental dogs were weighed daily before and up to 30 days after surgery.

Blood samples were collected on Days 0,7 and 21 after surgery. Serum was extracted and stored at $-20^{\circ} \mathrm{C}$

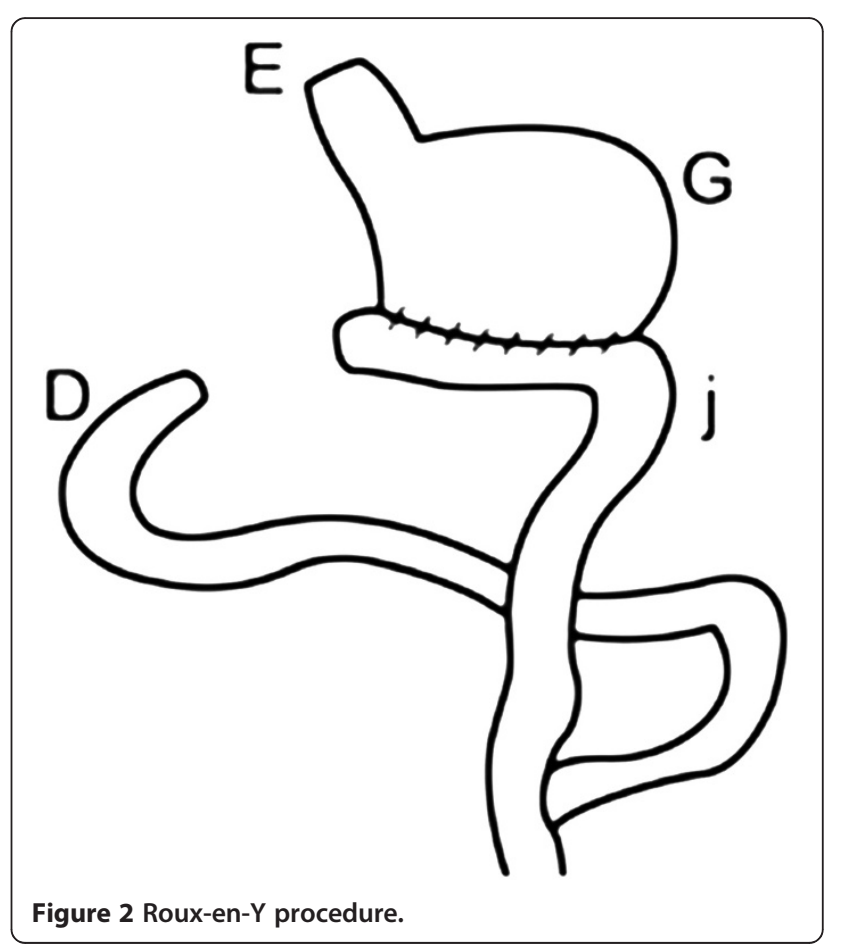




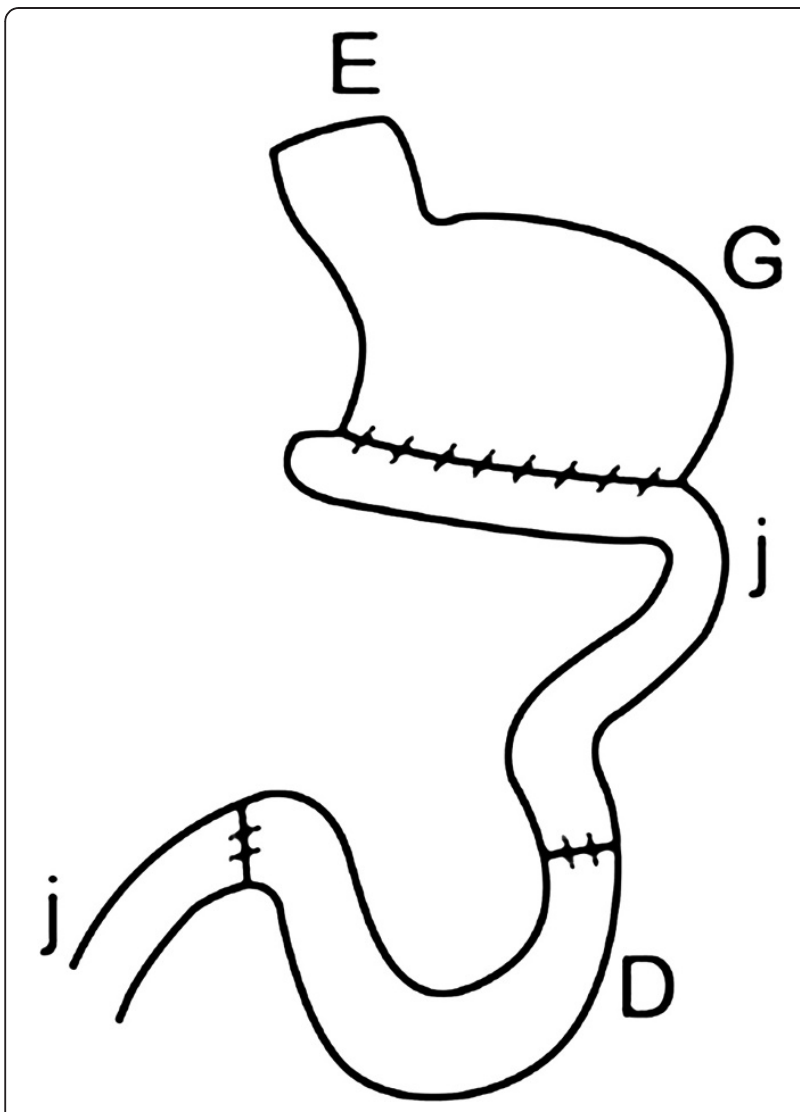

Figure 3 Jejunal Loop Interposition procedure.

until assayed. Urea concentration was measured using diacetylmonoxime reagent. Creatinine concentration was measured by the Jaffe reaction. Aspartate aminotransferase activity was measured by the direct combination of onalacetic acid with dinitrophenylhydraline and by the color change in an alkaline solution. Alkaline phosphatase activity was measured with p-nitrophenyl phosphate as a substrate. Total protein concentration was measured by the Biuret method. Triglyceride concentration was measured by the glycerol-phosphate onidase p-aminophenazone method. Cholesterol was measured by the cholesterol onidase p-aminophenazone method. Glucose concentration was measured by glucose onidase-aminophenazone method. Hexose concentration was measured by o-toluidine reagent. Sodium and potassium concentrations were determined by flame photometry. Fecal samples were taken on Day 28 after surgery and the quality of stool fat was estimated using Sudan black III method. All materials and kits of biochemical tests were purchased from Ziest Chemie Diagnostics, Iran.

One month after surgery, 6 serial positive contrast radiographs were taken at $5,10,20,40,60$ and 90 minutes after oral administration of barium sulfate (Daroupakhsh pharmaceutical, Iran) in order to determine the postoperative complications including leakage from the sites of anastomosis, narrowing, strictures or obstruction on the anastomotic sites, gastric emptying time and other abnormalities involving the small intestine.

Data were analyzed using GLM procedure with repeated measures included in the model. Single point measurements were tested using t-student test and Kruskal-Wallis test.

\section{Results}

All dogs recovered from anesthesia with no complications during the surgery and recovery. There was no evidence of wound infection or dehiscence in patients. Dogs started to eat on Day 4 after surgery without any sign of regurgitation. No serious postoperative complications were observed. There were no signs of premature vasomotor disturbances, such as dizziness, faintness, weakness and alimentary disorders. One dog in Rouxen-Y group showed nausea, regurgitation and vomiting and received conservative therapy.

Body weights before the operation were similar between groups (Roux-en-Y: $26.5 \pm 2.04 \mathrm{~kg}$; Jejunal loop interposition: $26.5 \pm 1.5 \mathrm{~kg} ; \mathrm{P}>0.05$ ). There was gradual reduction

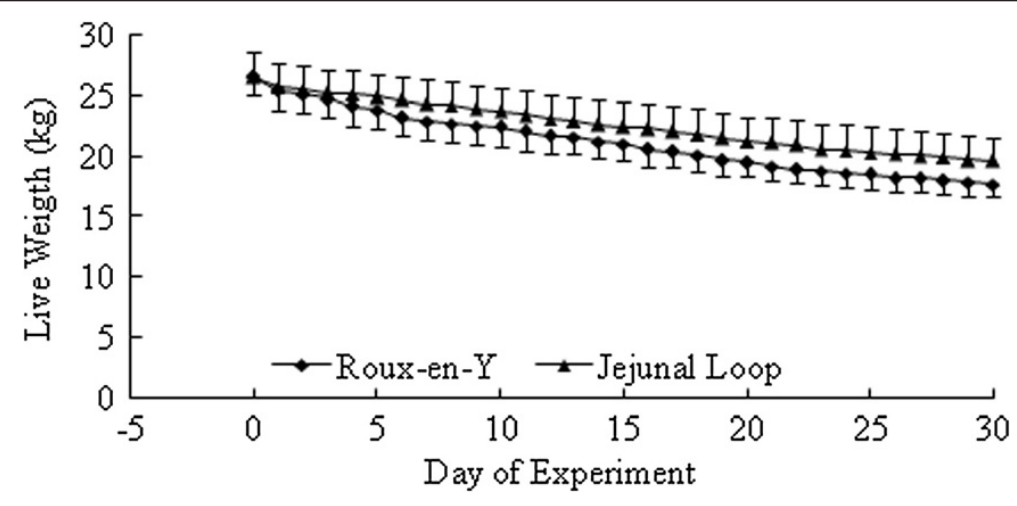

Figure 4 Body weight reduction until one month after surgery in both groups. 
in body weight after surgery in both groups (Figure 4). Body weight one month after surgery reached $17.7 \pm 1.98 \mathrm{~kg}$ and $19.6 \pm 1.09 \mathrm{~kg}$ in Roux-en-Y and Jejunal loop interposition, respectively. In this study, 30 days after surgery, all experimental animals have shown significant weight loss but the difference between two groups was not significant. Fecal fat was observed in animals after Roux-enY operation but not after Jejunal Loop interposition.

Blood parameters, including Glucose, Hexose, TC, TG, TG/TC, Total protein, Albumin, Globulin, Urea, BUN, Creatinin, BUN/Creatinin, Fe, SGOT, SGPT, ALP. CL, Na and $\mathrm{K}$ were not different between experimental groups (Table 1; P > 0.05).

Radiographic findings, conducted for 3 days after surgery, did not reveal any signs of anatomic abnormality, organ displacement, anastomotic leakage and narrowing or obstruction at the sites of the anastomosis. One month after surgery, radiographic findings did not show any signs of leakage or strictures at the anastomotic sites (Figure 5). Slight abnormal jejunal movements were observed in 2 dogs in Roux-en-Y and one dog in Jejunal loop interposition. Gastric emptying time was $89 \pm 7.4$ minutes in Roux-en-Y and $86 \pm 4.1$ minutes in Jejunal loop interposition $(P>0.05)$. There was no sign of narrowing, stricture or obstruction in the anastomotic sites.

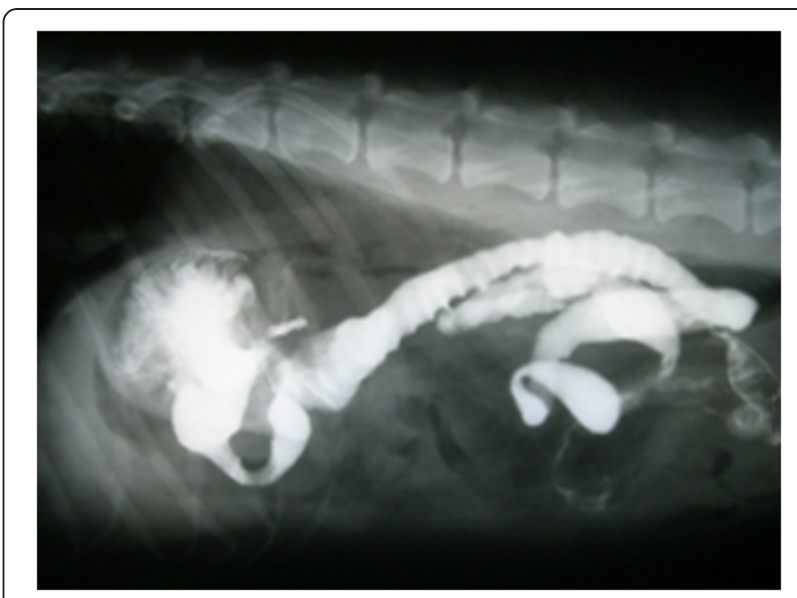

Figure 5 Positive contrast radiographs showing more jejunal movement than normal without any signs of leakage, strictures and other abnormalities.

\section{Discussion}

Present study was conducted to compare Roux-en-Y and Jejunal Loop interposition reconstructive techniques for subtotal gastrectomy using laparoscopic assisted surgery in dog. All dogs recovered following operation without any particular complications. This could be due to the

Table 1 Biochemical parameters of two experimental groups (Roux-en-Y and Jejunal Loop interposition using assisted laparoscopy) on Day 1, 7 and 21 after subtotal gastrectomy in dogs

\begin{tabular}{|c|c|c|c|c|c|c|c|}
\hline \multirow[t]{2}{*}{ Parameters } & \multirow{2}{*}{$\begin{array}{l}\text { Groups } \\
\text { Days }\end{array}$} & \multicolumn{3}{|c|}{ Roux-en-Y } & \multicolumn{3}{|c|}{ Jejunal loop interposition } \\
\hline & & 1 & 7 & 21 & 1 & 7 & 21 \\
\hline \multicolumn{2}{|c|}{ Glucose(mm/dl) } & $4.1 \pm 1.54$ & $3.7 \pm 0.27$ & $3.6 \pm 0.49$ & $4.9 \pm 0.66$ & $4.7 \pm 0.63$ & $4.9 \pm 0.52$ \\
\hline \multicolumn{2}{|c|}{ Hexose (mg/dl) } & $70.7 \pm 34.24$ & $58.9 \pm 4.47$ & $57.1 \pm 11.05$ & $104.6 \pm 15.09$ & $87.9 \pm 9.35$ & $96.9 \pm 7.98$ \\
\hline \multicolumn{2}{|c|}{ Total Cholestrol (mm/l) } & $2.9 \pm 0.32$ & $3.1 \pm 0.83$ & $1.8 \pm 0.41$ & $2.8 \pm 0.32$ & $1.6 \pm 0.11$ & $1.5 \pm 0.05$ \\
\hline \multicolumn{2}{|c|}{ Three Glyceride(mm/l) } & $0.5 \pm 0.12$ & $0.4 \pm 0.07$ & $0.3 \pm 0.07$ & $0.4 \pm 0.14$ & $0.3 \pm 0.06$ & $0.4 \pm 0.09$ \\
\hline \multicolumn{2}{|l|}{$\mathrm{Tg} / \mathrm{Tc}$} & $0.4 \pm 0.06$ & $0.4 \pm 0.24$ & $0.5 \pm 0.04$ & $0.4 \pm 0.16$ & $0.5 \pm 0.11$ & $0.6 \pm 0.15$ \\
\hline \multicolumn{2}{|c|}{ Total Protein(g/dl) } & $8.7 \pm 1.07$ & $6.9 \pm 0.37$ & $7.4 \pm 0.34$ & $6.7 \pm 0.31$ & $5.9 \pm 0.22$ & $7.1 \pm 0.68$ \\
\hline \multicolumn{2}{|c|}{ Albumin(mg/dl) } & $5.9 \pm 0.52$ & $5.6 \pm 0.14$ & $5.1 \pm 0.23$ & $5.6 \pm 0.32$ & $5.3 \pm 0.35$ & $5.2 \pm 0.29$ \\
\hline \multicolumn{2}{|c|}{ Globulin(mg/dl) } & $2.7 \pm 0.58$ & $1.3 \pm 0.32$ & $2.3 \pm 0.41$ & $1.1 \pm 0.12$ & $0.5 \pm 0.12$ & $1.8 \pm 0.82$ \\
\hline \multicolumn{2}{|l|}{ Urea $(\mathrm{mm} / \mathrm{l})$} & $6.5 \pm 0.72$ & $4.5 \pm 0.56$ & $5.2 \pm 0.27$ & $5.8 \pm 0.91$ & $5.8 \pm 1.37$ & $4.7 \pm 0.75$ \\
\hline \multicolumn{2}{|l|}{ BUN(mm/l) } & $6.6 \pm 0.72$ & $4.5 \pm 0.56$ & $5.2 \pm 0.27$ & $5.8 \pm 0.91$ & $5.8 \pm 1.37$ & $4.7 \pm 0.75$ \\
\hline \multicolumn{2}{|c|}{ Creatinine(mg/dl) } & $3.4 \pm 0.62$ & $2.3 \pm 0.11$ & $2.6 \pm 0.14$ & $2.6 \pm 0.38$ & $2.7 \pm 0.38$ & $3.2 \pm 0.36$ \\
\hline \multicolumn{2}{|c|}{ BUN/Creatinine } & $5.6 \pm 0.86$ & $5.5 \pm 0.86$ & $5.5 \pm 0.15$ & $6.1 \pm 0.24$ & $5.8 \pm 1.16$ & $4.2 \pm 1.02$ \\
\hline \multicolumn{2}{|l|}{$\mathrm{Fe}(\mathrm{um} / \mathrm{l})$} & $40.4 \pm 11.72$ & $15.7 \pm 6.86$ & $16.3 \pm 4.42$ & $15.01 \pm 0.88$ & $28.2 \pm 2.51$ & $15.9 \pm 2.01$ \\
\hline \multicolumn{2}{|l|}{ SGOT(lu/l) } & $234 \pm 71.02$ & $38.6 \pm 26.52$ & $54 \pm 14$ & $104.3 \pm 4.33$ & $90.5 \pm 24.66$ & $96.9 \pm 58.74$ \\
\hline \multicolumn{2}{|l|}{ SGPT(Iu/I) } & $10.5 \pm 5.37$ & $3.4 \pm 2.32$ & $4.9 \pm 1.04$ & $20.2 \pm 11.83$ & $3.7 \pm 1.41$ & $9.2 \pm 5.23$ \\
\hline \multicolumn{2}{|l|}{ ALP(u/l) } & $106.8 \pm 64.08$ & $244.3 \pm 125.28$ & $74.3 \pm 29.02$ & $102.9 \pm 75.37$ & $70.4 \pm 2.45$ & $111.4 \pm 79.46$ \\
\hline \multicolumn{2}{|l|}{$\mathrm{Cl}(\mathrm{mmol} / \mathrm{l})$} & $137.1 \pm 2.97$ & $131.1 \pm 5.95$ & $134.7 \pm 5.67$ & $128.1 \pm 4.24$ & $138.2 \pm 2.59$ & $128.1 \pm 2.97$ \\
\hline \multicolumn{2}{|l|}{$\mathrm{Na}(\mathrm{mmol} / \mathrm{l})$} & $159.3 \pm 6.33$ & $160 \pm 4.93$ & $156 \pm 1.52$ & $160 \pm 4.16$ & $159.3 \pm 6.64$ & $162.3 \pm 2.33$ \\
\hline \multicolumn{2}{|l|}{$\mathrm{K}(\mathrm{mmol} / \mathrm{l})$} & $5.8 \pm 0.55$ & $6.3 \pm 0.28$ & $6.03 \pm 0.14$ & $6.1 \pm 0.34$ & $5.9 \pm 0.44$ & $6.3 \pm 0.26$ \\
\hline
\end{tabular}


lesser inflammatory response of the gut and lesser bacterial and endotoxin release occurring following laparoscopic assisted gasterectomy [17].

The alterations in body weights were similar before and after operation between experimental groups. One month after surgery, animals in both groups have shown significant weight loss. Similarly, in most studies, significant body weight loss after gastrectomy was noticed $[1,2,7,22-24]$. In one study with ten patients, the average weight loss was $25 \mathrm{~kg}$ [23]. In another study, 24\% body weight loss was noted in a group of 16 patients and only one-third of patients were able to achieve their ideal body weight after surgery [25]. Although the significant weight loss is a consequence of gastric resection, the cause of the malnutrition is still controversial. Malabsorption due to bacterial overgrowth, small intestinal mucosal lesions, pancreatic enzymatic deficiency and decrease of food passing time may be considered as part causes of malnutrition following gastric resection [26-28]. Insufficient caloric intake due to loss of appetite, intestinal motility alteration and early satiety may be also considered as other causes of malnutrition following gastrectomy [23,28,29].

In the present study, fecal fat was observed in animals after Roux-en-Y operation but not after Jejunal Loop interposition. Previously, it was reported that esophagojejunostomy did not affect fecal fat; while, fecal fat was higher in Roux-en-Y esophagojejunostomy group than esophagojejunoduodenostomy group [28]. The absorption of carbohydrate in the GI tract is high after gastrectomy, whether the duodenum was bypassed or not. In case of normal duodenal food passage, the rate of absorption for protein and fat after operation were $85 \%$ and 93\%, respectively; whereas, this was slightly lower in duodenal bypass cases [30]. This illustrates the importance of the duodenal passing role in food absorption, especially for fat [28].

Concentrations of blood glucose were not different between experimental groups. Some disturbance in blood glucose concentrations were observed following gastrectomy [23]. Type of reconstructive method affects glucose level. Roux-en-Y and pouch construction and duodenal exclusion approaches were associated with high glucose levels; however, by preserving the duodenal route the pathologic glucose tolerance did not develop [31]. In a gastrectomized patient with preservation of the duodenum, a higher level of glucose was reported during the first 45 minutes after a liquid test meal [32]. Concentrations of glucose in patients with duodenal exclusion (Roux-en-Y) were higher than that of the control group; this, in turn, supports the hypothesis that exclusion of duodenal passage disturbs glucose homeostasis [33]. In the present study, the concentrations of blood glucose were in the normal range. The amount of nitrogen losses in fecal matter can be increased after gastrectomy which indicates a decrease in the quantity of ingested protein [22]. The rate of amino acid absorption in gastrectomized animals is rapid [22,25]; but the efficiency of absorption is decreased resulting in the increase of nitrogen losses through defecation. The rate of nitrogen losses in animals with a normal duodenum is less than with duodenal bypass [22].

Radiographic findings, conducted for 3 days after surgery, did not reveal any signs of anatomic abnormality, organ displacement, anastomotic leakage and narrowing or obstruction at the sites of the anastomosis. One month after surgery, radiographic findings did not show any signs of leakage or strictures at the anastomotic sites. The change in the peristaltic activity of the jejunum can lead to problematic delayed gastric emptying time. after Roux-en-Y gastrojejunostomy and occurs in $25 \%$ to $30 \%$ of patients [34]. Different types of reconstructive techniques may have different results in transit of the solid meal [7]. Gastric emptying time was less than 100 minutes and similar between experimental groups. The mean operating time was $117 \pm 11.3 \mathrm{~min}$ in Roux-en-Y and $116 \pm 16.9$ min in Jejunal loop interposition [35]. In other studies in humans, mean operating time of 225 minutes for laparoscopic total gastrectomy [18] and $72 \pm 4$ minutes for laparoscopic assisted distal gastrectomy [17] were reported. Anastomotic leakage is one of the most important and early complications following total or partial gastrectomy, especially in esophagojejunal anastomosis because of poor blood supply to the esophagus [36]. About $35-65 \%$ of all operative and postoperative deaths are due to anastomotic breakdown and leakage [37]. In the present study, there was no sign of narrowing, stricture or obstruction in the anastomotic sites.

\section{Conclusion}

Considering the fact that the power of the test might be low due to low number of animals in each experimental group; however, both Roux-en-Y and Jejunal loop interposition might be considered as suitable reconstructive techniques following gastrectomy with no particular difference in clinical and para-clinical parameters and postoperative complications in dogs. Further investigations with a larger number of animals and longer postoperative observation may be warranted based on the findings of the present study.

\section{Competing interests}

The authors declare that they have no competing interests.

\section{Authors' contributions}

$J B, M A$ and ARK designed and performed the study. FA conducted the biochemistry analysis. JB drafted the manuscript. ANN performed the statistical analysis and helped in interpreting the data and drafting the manuscript. All authors read and approved the final manuscript. 


\section{Acknowledgements}

The authors wish to thank the deputy of Research of the Faculty of Veterinary Medicine, University of Tehran and Center of Excellence for Veterinary Research on Iranian Indigenous Animals for providing financial assistance to complete this research. We also express our great appreciations to Dr William Aspden for his kind assistance in editing the manuscript.

\section{Author details}

${ }^{1}$ Faculty of Veterinary Medicine, University of Tehran, Tehran, Iran. ${ }^{2}$ Department of Surgery, Faculty of Medicine, Shahed University, Tehran, Iran. ${ }^{3}$ Minimally Invasive Surgery Research Centre, Rasool Akram Hospital, Tehran University of Medical Sciences, Tehran, Iran. ${ }^{4}$ Department of Surgery and Radiology, Faculty of Veterinary Medicine, University of Tehran, P. O. Box: 14155-6453, Tehran, Iran.

Received: 14 September 2012 Accepted: 21 November 2012 Published: 11 December 2012

\section{References}

1. Scurtu R, Groza N, Otel O, Goia A, Funariu G: Quality of life in patients with esophagojejunal anastomosis after total gastrectomy for cancer. Romanian J Gastroenterol 2005, 14:367-372.

2. Qin $X Y$, Lei $Y$, Liu FL: Effects of two methods of reconstruction of digestive tract after total gastrectomy on gastrointestinal motility in rats. World J Gastroenterol 2003, 9:1051-1053.

3. Sakamoto T, Fujimaki M, Tazawa K: lleocolon interposition as a substitute stomach after total or proximal gastrectomy. Ann Surg 1997, 226:139-145.

4. Zherlov G, Koshel A, Orlova Y, Zykov D, Skolov S, Rudaya N, Karpovitch A New type of jejunal interposition method after gastrectomy. World J Surg 2006, 30:1475-1480.

5. Csendes A, Burgos AM, Smok G, Burdiles P, Braghetto I, Díaz JC: Latest results (12-21 Years) of a prospective randomized study comparing Billroth II and Roux-en-Y anastomosis after a partial gastrectomy plus vagotomy in patients with duodenal ulcers. Ann Surg 2009, 249:189-194.

6. Tono C, Terashima M, Takagane A, Abe K: Ideal reconstruction after total gastrectomy by the interposition of a jejunal pouch considered by emptying time. World J Surg 2003, 27:1113-1118.

7. Miholic J, Meyer HJ, Kotzerke J, Balks J, Aebert H, Jahne J, Weimann A, Pichlmayr R: Emptying of the gastric substitute after total gastrectomy jejunal interposition versus roux-y esophagojejunostomy. Ann Surg 1989, 210:165-172.

8. Omori T, Nakajima K, Endo S, Takahashi T, Hasegawa J, Nishida T: Laparoscopically assisted total gastrectomy with jejuna pouch interposition. Surg Endosc 2006, 20:1497-1500.

9. Mathias JR, Fernandez A, Sninsky CA, Clench MH, Davis RH: Nausea, vomiting and abdominal pain after Roux-en-Y anastomosis: motility of the jejunal limb. Gastroenterol 1985, 88:101-107.

10. Pan Y, Li Q, Wang DC, Wang JC, Liang H, Liu JZ, Cui QH, Sun T, Zhang RP, Kong DL, Hao XS: Beneficial effects of jejunal continuity and duodenal food passage after total gastrectomy: a retrospective study of 704 patients. European J Surg Oncol 2008, 34:17-22.

11. Van der Mijle HC, Kleibeuker JH, Limburg AJ, Bleichrodt RP, Beekhuis H, van Schilfgaarde R: Manometric and scintigraphic studies of the relation between motility disturbances in the Roux limb and the Roux-en-Y syndrome. Am J Surg 1993, 166:11-17.

12. Bassotti G, Chiarinelli ML, Germani U, Chiarioni G, Morelli A: Effect of some abdominal surgical operations on some bowel motility in humans: our experience. J Clin Gastroenterol 1995, 21:211-216.

13. Ye ZY, Sun YS, Zhang Q, Wang YY, Dai QQ, Shao QS, Zhang $W, L u ̈ Z Y, X u J$ : Different procedures of alimentary tract reconstruction after subtotal distal gastrectomy in dogs. Zhonghua Yi Xue Za Zhi 2010, 90:2704-2707.

14. Ishigami S, Natsugoe S, Hokita S, Aoki T, Kashiwagi H, Hirakawa K, Sawada T, Yamamura Y, Itoh S, Hirata K, Ohta K, Mafune K, Nakane Y, Kanda T, Furukawa H, Sasaki I, Kubota T, Kitajima M, Aikou T: Postoperative longterm evaluation of interposition reconstruction compared with Roux-en$\mathrm{Y}$ after total gastrectomy in gastric cancer: prospective randomized controlled trial. Am J Surg 2011, 202:247-253.

15. Nakane $Y$, Michiura $T$, Inoue $K$ : A randomized clinical trial of pouch reconstruction after total gastrectomy for cancer: which is the better technique, Roux-en-Y or interposition? Hepatogastroenterol 2001, 48:903-907.
16. Iwahashi M, Nakamori M, Nakamura M: Evaluation of double tract reconstruction after total gastrectomy in patients with gastric cancer: prospective randomized controlled trial. World J Surg 2009, 33:1882-1888.

17. Hiki N, Shimizu N, Yamaguchi H, Imamura K, Kami K, Kubota K, Kaminishi M: Manipulation of the small intestine as a cause of the increased inflammatory response after open compared with laparoscopic surgery. British J Surg 2006, 93:195-204.

18. Shehzad K, Mohiuddin K, Nizami S, Sharma H, Khan IM, Memon B, Memon MA: Current status of minimal access surgery for gastric cancer. Surg Oncol 2007, 16:85-98.

19. Abdi M, Bakhtiari J, Golfam F, Khalaj A, Masoudifar M, Bahonar A Comparative evaluation of laparoscopic assisted gastrectomy versus open surgery using Roux-en-Y reconstruction technique in dogs. J Vet Res 2011, 66:123-130.

20. Huscher C, Mingoli A, Sgarzini G, Sansonetti A, Paola MD, Recher A, Ponzano C: Laparoscopic versus open subtotal gastrectomy for distal gastric cancer, five-year results of a randomized prospective trial. Ann Surg 2005, 241:232-237.

21. Kim MC, Choi HJ, Jung GJ, Kim HH: Techniques and complications of laparoscopy-assisted distal gastrectomy (LASG) for gastric cancer. European J Surg Oncol 2007, 33:700-705

22. Mccorkle HJ, Harper HA: The problem of nutrition following complete gastrectomy. Ann Surg 1954, 140:467-473.

23. Bradly EL, Issacs J, Hersh T, Davidson ED, Millikan W: Nutritional consequences of total gastrectomy. Ann Surg 1975, 182:415-428.

24. Sweet $\mathrm{RH}$ : Total gastrectomy by the transthoracic approch, a subsequent report. Ann Surg 1953, 138:297-310.

25. Kelly WD, MacLean LD, Perry JF: A study of patients following total and near-total gastrectomy. Surg 1954, 35:964-982.

26. Adams JF: The clinical and metabolic consequences of total gastrectomy III. Notes on metabolic functions, deficiency states, changes in intestinal histology and radiology. Scand J Gastroenterol 1968, 3:152-159.

27. Lundh G: Intestinal digestion and absorption after gastrectomy. Acta Chir Scand Suppl 1955, 231:1-82.

28. Min JS, Choi SH, Noh SH, Kim MW: Nutritional effects according to reconstructional methods after total gastrectomy. Yonsei Med J 1995, 36:9-14.

29. Braga M, Zuliani W, Foppa L, Carlo VD, Cristallo M: Food intake and nutritional status after total gastrectomy: results of a nutritional follow up. Br J Surg 1988, 75:477-480.

30. Sakakibara N, Ogawa G: Carcinoma of the cardia: interposed transplantation jejunal following radical total gastrectomy. In Color Atlas of Gastrointestinal Surgery. 1989:189-207.

31. Schwarz A, Buchler M, Usinger $K$, Rieger $H$, Glasbrenner B, Friess $H$, Kunz R, Beger HG: Importance of the duodenal passage and pouch volume after total gastrectomy and reconstruction with the UIm pouch: prospective randomized clinical study. World J Surg 1996, 20:60-66.

32. Friess $H$, Bohm J, Muller MW, Glasbrenner B, Riepl RL, Malfertheiner $P$, Buchler MW: Maldigestion after total gastrectomy is associated with pancreatic insufficiency. Am J Gastroenterol 1996, 91:341-347.

33. Kalmar K, Nemeth J, Kelemen G, Horva'th OP: Postprandial gastrointestinal hormone production is different, depending on the type of reconstruction following total gastrectomy. Ann Surg 2006, 243:465-471.

34. Gowen GF: Delayed gastric emptying after roux-en-y due to four types of partial obstruction. Ann Surg 1992, 215:363-367.

35. Yakoub D, Athanasiou T, Tekkis P, Hanna GB: Laparoscopic assisted distal gastrectomy for early gastric cancer: Is it an alternative to the open approach? Surg Oncol 2009, 18:322-333.

36. Walther B, Strand SE, Oscarson J, Lowenhielm P, Stahlberg F, Evander A, Uvelius B: Healing of esophagojejunal anastomoses after experimenta total gastrectomy, a comparative study using manually sutured or stapled anastomoses. Ann Surg 1986, 203:439-446.

37. Levine MS, Fisher AR, Rubesin SE, Laufer I, Herlinger H, Rosato EF: Complications after total gastrectomy and esophagojejunostomy: radiologic evaluation. Am J Roentgenol 1991, 157:1189-1194.

\section{doi:10.1186/1756-0500-5-679}

Cite this article as: Bakhtiari et al: Comparative evaluation of two reconstructive methods following laparoscopic assisted subtotal gastrectomy in dogs. BMC Research Notes 2012 5:679. 